\title{
Chocolate consumption and risk of gestational diabetes mellitus: the Japan Environment and Children's Study
}

\author{
Jia-Yi Dong ${ }^{1}$, Takashi Kimura ${ }^{1}$, Satoyo Ikehara ${ }^{1}$, Meishan Cui ${ }^{1}$, Yoko Kawanishi ${ }^{2}$, Kazumasa Yamagishi ${ }^{3}$, \\ Kimiko Ueda ${ }^{4}$, Hiroyasu Iso ${ }^{1,3 *}$ and the Japan Environment and Children's Study Group \\ ${ }^{1}$ Public Health, Department of Social Medicine, Osaka University Graduate School of Medicine, Osaka, Japan \\ ${ }^{2}$ Department of Obstetrics and Gynecology, Osaka University Graduate School of Medicine, Osaka, Japan \\ ${ }^{3}$ Department of Public Health Medicine, Faculty of Medicine, University of Tsukuba, Tsukuba, Japan \\ ${ }^{4}$ Maternal \& Child Health Information Center, Osaka Women's and Children's Hospital, Osaka, Japan
}

(Submitted 20 March 2019 - Final revision received 4 July 2019 - Accepted 18 July 2019)

\section{Abstract}

The association of chocolate consumption with risk of gestational diabetes has not been examined. We aimed to investigate the prospective association between chocolate consumption and risk of gestational diabetes in a large birth cohort in Japan. A total of 97454 pregnant women with a median gestational age of 12 weeks were recruited from January 2011 to March 2014. Data on demographic information, disease history, socio-economic status, lifestyle and dietary habits were obtained at the study enrolment. Dietary intake during the past 12 months before study enrolment was assessed through a semi-quantitative FFQ. The logistic regression was used to obtain the OR of gestational diabetes in relation to chocolate consumption. Among 84948 women eligible for the analysis, 1904 cases of gestational diabetes (2.2\%) were identified during the period of pregnancy. After controlling for potential confounding factors including age, smoking status, drinking status, education level, occupation, pre-pregnant BMI, depression, previous history of macrosomia babies, parity, physical activity and dietary factors, women in the highest quartile of chocolate consumption, compared with those in the lowest quartile, had a significantly lower risk of developing gestational diabetes (OR $0.78,95 \%$ CI $0.67,0.90 ; P$ for trend $=0.002$ ). Stratified analyses suggested that the association was not significantly modified by prepregnancy BMI, age, parity, smoking status or drinking status. The present prospective cohort study provided evidence that chocolate consumption was associated with a significant lower risk of gestational diabetes in Japanese women.

Key words: Gestational diabetes: Chocolate: Prospective cohort studies: Prevention: Epidemiology

Gestational diabetes mellitus (GDM), defined as glucose intolerance with onset or first recognition during pregnancy, has a number of adverse health consequences in both mothers and their offspring ${ }^{(1,2)}$. Women with a history of GDM are at substantially high risk of developing type 2 diabetes in later life ${ }^{(3)}$. A recent pooled analysis of more than 2 million women found that women with a previous history of GDM, compared with those without it, had a 7.7-fold risk of type 2 diabetes $^{(4)}$. Emerging evidence also showed that GDM significantly increased the risk of CVD in later life ${ }^{(5)}$. On the other hand, children of mother with a history of GDM have a higher risk to be overweight and develop insulin resistance ${ }^{(6,7)}$. It is, therefore, of importance to identify modifiable risk factors for primary prevention of GDM.

Chocolate, a popular food over the world, has received increasing interest recently. Many intervention studies have documented beneficial effects of chocolate or other cocoa products on improving blood pressure ${ }^{(8)}$, flow-mediated dilation ${ }^{(9)}$, lipid profile $^{(10)}$ and insulin resistance ${ }^{(10)}$. Observational studies have also showed that chocolate consumption was associated with decreased risk of type 2 diabetes, stroke, acute myocardial infarction and CVD death ${ }^{(11)}$. However, no study has examined the association between chocolate consumption and GDM. The present study, as the first one, aimed to examine the prospective association between chocolate consumption and the risk of GDM in a large birth cohort of Japanese women.

\section{Subjects and methods}

The Japan Environment and Children's Study, launched by the Ministry of Environment, is an ongoing national birth cohort study examining the effects of environmental factors along with genetic, social and lifestyle-related factors on children health and development from the fetal period until 13 years old. The detail of study design has been described elsewhere ${ }^{(12,13)}$. Briefly, after excluding 5645 pregnancies that were not the first enrolment from 103099 pregnancies, 97454 pregnant women with a

Abbreviation: GDM, gestational diabetes mellitus.

* Corresponding author: H. Iso, email iso@pbhel.med.osaka-u.ac.jp 


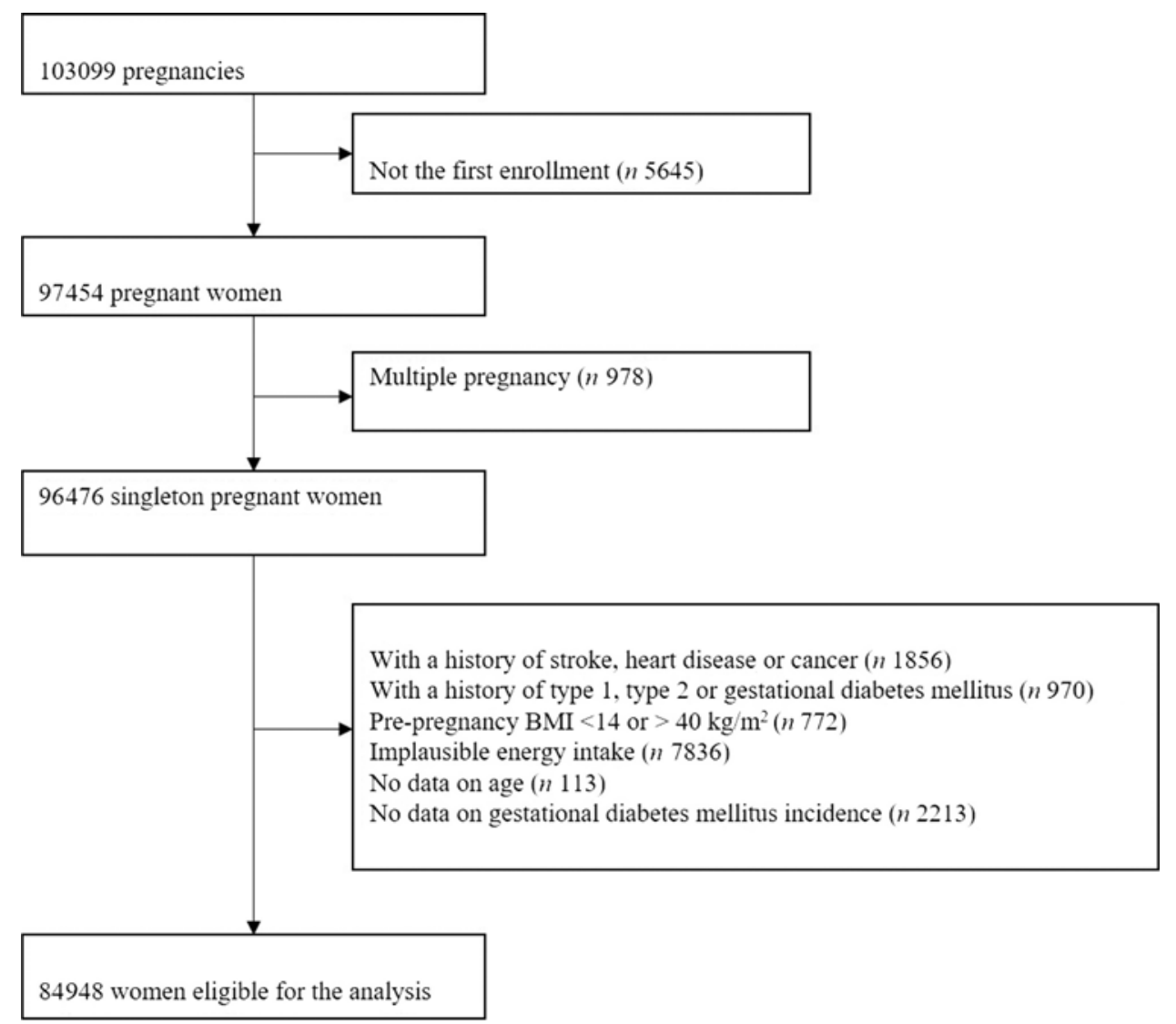

Fig. 1. Flow chart for participant selection.

median gestational age of 12 weeks were recruited in fifteen urban and rural areas across Japan from January 2011 to March 2014. A self-administered questionnaire was used to collect the baseline data on demographic information, disease history, socio-economic status, lifestyle and dietary habits of each mother at study enrolment.

Women were eligible for the analysis if they had a singleton pregnancy and had no history of stroke, heart disease, cancer, type 1 diabetes, type 2 diabetes or GDM at study enrolment and provided essential data for the analysis. Women were excluded if they had an extreme BMI (calculated by self-reported height and weight) before pregnancy (i.e. $<14$ or $>40 \mathrm{~kg} / \mathrm{m}^{2}$ ) or reported an implausible total energy intake (upper $2.5 \%$ and lower $2.5 \%$ ). Finally, a total of 84948 women were included in the present analysis. Fig. 1 shows the flow chart for participant selection.

Dietary intake was assessed using a semi-quantitative FFQ (172 items) which has been used and validated in another large Japanese cohort study ${ }^{(14)}$. Participants were asked about the frequency and portion size of chocolate consumption as well as other food and beverage intake during the past 12 months before they were recruited. The predefined frequency categories ranged from $<$ once/month to $\geq 7$ times/d for food items and from $<1$ cup/week to $\geq 10 \mathrm{cups} / \mathrm{d}$ for beverage items. The quantity of chocolate consumption was computed by multiplying the intake frequency by the portion size. The portion size of chocolate was
$25 \mathrm{~g}$ in one time, and two additional options are less than half or more than 1.5 times in one time. Sweet pastry and snack bars were not included as chocolate. Total dietary energy as well as other nutrient intake was calculated based on the fifth revised edition of the Standard Tables of Food Composition in Japan.

The outcome of the present study was the first incidence of GDM. Medical record transcripts, performed after delivery by physicians, midwives, nurses and/or research coordinators, were used to identify GDM cases. Cases were diagnosed using the 75-g oral glucose tolerance test, according to the criteria of the Japan Diabetes Society ${ }^{(15)}$.

The present study was based on the data set of jecsag-20160424, which was released in June 2016 and revised in October 2016. This data set included information of baseline characteristics, lifestyle during pregnancy, pregnancy outcomes and health examination at 1 month after giving birth. Age-adjusted means and proportions of the characteristics of pregnant women were calculated according to chocolate consumption. Generalised linear models were used for trend test. We used the logistic regression to obtain the OR of GDM associated with chocolate consumption because GDM requires the fact of being pregnant and does not depend on time. The lowest intake group was treated as the reference. All OR were age adjusted in model 1 . We also adjusted for potential confounding factors of GDM in model 2, including smoking status (never, past and current smokers), drinking status (never, past and current 
Table 1. Baseline characteristics of 84948 singleton pregnant women according to chocolate consumption* (Mean values and standard deviations; numbers of participants; percentages)

\begin{tabular}{|c|c|c|c|c|c|c|c|c|c|}
\hline & \multicolumn{2}{|c|}{ Q1 } & \multicolumn{2}{|c|}{ Q2 } & \multicolumn{2}{|c|}{ Q3 } & \multicolumn{2}{|c|}{ Q4 } & \multirow[b]{2}{*}{$P$ for trend } \\
\hline & Mean & SD & Mean & SD & Mean & SD & Mean & SD & \\
\hline Median intake (g/week) & \multicolumn{2}{|c|}{0} & \multicolumn{2}{|c|}{$11 \cdot 7$} & \multicolumn{2}{|c|}{$37 \cdot 5$} & \multicolumn{2}{|c|}{$87 \cdot 5$} & \\
\hline Participants $(n)$ & \multicolumn{2}{|c|}{21579} & \multicolumn{2}{|c|}{23922} & \multicolumn{2}{|c|}{19093} & \multicolumn{2}{|c|}{20354} & \\
\hline Age (years) & 30.9 & $5 \cdot 1$ & $30 \cdot 6$ & $5 \cdot 1$ & $30 \cdot 7$ & $5 \cdot 0$ & 30.5 & 4.9 & $<0.001$ \\
\hline BMI before pregnancy $\left(\mathrm{kg} / \mathrm{m}^{2}\right)$ & $21 \cdot 3$ & $3 \cdot 3$ & $21 \cdot 3$ & $3 \cdot 2$ & $21 \cdot 1$ & 3.0 & $21 \cdot 0$ & $3 \cdot 0$ & $<0.001$ \\
\hline Depression (\%) & \multicolumn{2}{|c|}{0.4} & \multicolumn{2}{|c|}{0.3} & \multicolumn{2}{|c|}{0.2} & \multicolumn{2}{|c|}{0.3} & $<0.001$ \\
\hline History of macrosomia babies (\%) & \multicolumn{2}{|c|}{0.5} & \multicolumn{2}{|c|}{0.5} & \multicolumn{2}{|c|}{0.5} & \multicolumn{2}{|c|}{0.5} & 0.90 \\
\hline Nulliparous (\%) & \multicolumn{2}{|c|}{$39 \cdot 7$} & \multicolumn{2}{|c|}{$41 \cdot 7$} & \multicolumn{2}{|c|}{$42 \cdot 3$} & \multicolumn{2}{|c|}{$43 \cdot 7$} & $<0.001$ \\
\hline Never smoker (\%) & \multicolumn{2}{|c|}{$55 \cdot 6$} & \multicolumn{2}{|c|}{$58 \cdot 9$} & \multicolumn{2}{|c|}{$60 \cdot 7$} & \multicolumn{2}{|c|}{$58 \cdot 7$} & $<0.001$ \\
\hline Never drinker (\%) & & & & & & & & & $<0.001$ \\
\hline Physical activity (MET-h/d) & 3.9 & $9 \cdot 6$ & 3.8 & $7 \cdot 8$ & 3.9 & 8.5 & $4 \cdot 0$ & $8 \cdot 3$ & $0 \cdot 10$ \\
\hline University or higher education (\%) & & & & & & & & & 0.30 \\
\hline Housewife (\%) & & & & & & & & & $<0.01$ \\
\hline Household income <2 million Yen (\%) & & & & & & & & & 0.07 \\
\hline Carbohydrate (\% of energy) & $56 \cdot 2$ & & $55 \cdot 7$ & & $55 \cdot 0$ & & $54 \cdot 1$ & & $<0.001$ \\
\hline Protein ( $\%$ of energy) & $13 \cdot 8$ & & $13 \cdot 6$ & & $13 \cdot 6$ & & $13 \cdot 1$ & & $<0.001$ \\
\hline Fat (\% of energy) & $28 \cdot 1$ & & 28.9 & & $29 \cdot 8$ & & $31 \cdot 3$ & & $<0.001$ \\
\hline SFA (\% of energy) & 8.6 & & 8.9 & & $9 \cdot 4$ & & $10 \cdot 3$ & & $<0.001$ \\
\hline MUFA (\% of energy) & $10 \cdot 5$ & & $10 \cdot 8$ & & $11 \cdot 1$ & & 11.5 & & $<0.001$ \\
\hline PUFA (\% of energy) & 5.9 & & 5.9 & & $6 \cdot 0$ & & $6 \cdot 1$ & & $<0.001$ \\
\hline Meat $(g / d)$ & $64 \cdot 8$ & $46 \cdot 1$ & $68 \cdot 9$ & $44 \cdot 8$ & $74 \cdot 3$ & 46.4 & $80 \cdot 6$ & $50 \cdot 6$ & $<0.001$ \\
\hline Red meat $(\mathrm{g} / \mathrm{d})$ & $36 \cdot 4$ & $31 \cdot 3$ & $38 \cdot .9$ & 30 & 41.7 & $31 \cdot 2$ & 44.9 & 33.5 & $<0.001$ \\
\hline Coffee $(g / d)$ & $92 \cdot 6$ & $158 \cdot 3$ & $100 \cdot 7$ & $160 \cdot 3$ & $107 \cdot 6$ & $150 \cdot 1$ & $124 \cdot 7$ & $176 \cdot 8$ & $<0.001$ \\
\hline Green tea $(g / d)$ & $159 \cdot 4$ & $262 \cdot 9$ & 156 & $239 \cdot 3$ & $161 \cdot 9$ & 236.5 & $178 \cdot 9$ & $268 \cdot 9$ & $<0.001$ \\
\hline $\operatorname{Milk}(g / d)$ & $122 \cdot 3$ & $247 \cdot 7$ & $120 \cdot 7$ & $225 \cdot 1$ & 125 & $223 \cdot 8$ & $125 \cdot 6$ & $215 \cdot 7$ & 0.04 \\
\hline Soya isoflavone $(\mathrm{mg} / \mathrm{d})$ & $29 \cdot 6$ & $29 \cdot 9$ & $29 \cdot 3$ & $27 \cdot 8$ & $31 \cdot 7$ & $26 \cdot 4$ & 33.4 & $27 \cdot 4$ & $<0.001$ \\
\hline $\mathrm{Mg}(\mathrm{mg} / \mathrm{d})$ & $211 \cdot 3$ & $94 \cdot 7$ & $219 \cdot 2$ & $88 \cdot 8$ & $239 \cdot 2$ & 86.9 & $264 \cdot 7$ & 94 & $<0.001$ \\
\hline Dietary fibre $(\mathrm{g} / 1000 \mathrm{~kJ})$ & $7 \cdot 5$ & $2 \cdot 7$ & $7 \cdot 3$ & $2 \cdot 4$ & $7 \cdot 4$ & $2 \cdot 2$ & $7 \cdot 5$ & $2 \cdot 2$ & $<0.001$ \\
\hline Snacks (potato chips and other crackers) $(\mathrm{g} / \mathrm{d})$ & $3 \cdot 1$ & 5.7 & 4.3 & $5 \cdot 2$ & 5.9 & 5.8 & 8.9 & 8.5 & $<0.001$ \\
\hline Total energy $(\mathrm{kJ} / \mathrm{d})$ & $6557 \cdot 6$ & $2176 \cdot 1$ & 6929.5 & $2093 \cdot 3$ & 7537.9 & 2099.9 & 8423.6 & $2307 \cdot 1$ & $<0.001$ \\
\hline
\end{tabular}

MET, metabolic equivalents.

* Generalised linear models were used for trend test.

drinker), education level (middle school, high school, junior or specialised training college, university or higher), occupation (fifteen categories), household income (nine categories), prepregnancy BMI (quintiles), depression (yes or no), previous history of macrosomia babies (yes or no), parity (0, 1, 2 or $\geq 3$ ) and physical activity (quintiles). In model 3, we further adjusted for dietary factors, including quintile intake of total meat, red meat, coffee, green tea, milk, soya isoflavone, $\mathrm{Mg}$, dietary fibre, dietary fat, saturated fat, snacks (potato chips and other crackers) and total energy. These dietary factors were selected from more than fifty kinds of foods and nutrients based on previous studies. We next conducted predefined subgroup analysis according to prepregnancy BMI, age, parity, smoking status and drinking status in order to examine whether these factors could modify the association between chocolate consumption and GDM. Interaction tests were conducted by entering the interaction terms in the logistical model. In addition, sensitivity analyses were carried out to test the robustness of the results by restricting the analysis in participants who reported no substantial change in their dietary habits during the past 5 years. We also repeated the analysis using energy-adjusted nutrients by the residual method and using quintiles for chocolate consumption. All analyses were performed using SAS software (version 9.4; SAS Institute Inc.). All $P$ values were two sided, and $P<0.05$ was considered as statistically significant.
The study protocol was reviewed and approved by the Ministry of the Environment's Institutional Review Board on Epidemiological Studies and by the Ethics Committees of all participating institutions. Written informed consent was obtained from all participants.

\section{Results}

The characteristics of pregnant women at study enrolment according to chocolate consumption are shown in Table 1. Median chocolate consumptions were $0,11.7,37.5$ and $87.5 \mathrm{~g} /$ week for the four quartiles. Compared with women in the lowest quartile of chocolate consumption, those in the highest quartile were younger, had a slightly lower BMI and were more likely to be nulliparous and use alcohol. In addition, higher chocolate consumption was also related to higher intake of total meat, red meat, coffee, green tea, soya isoflavone, dietary magnesium, dietary fat, snacks and total energy.

A total of 1904 (2.2\%) cases of GDM were identified from 84948 women during the period of pregnancy. In model 1, a basic model adjusting for age only, women in the highest quartile of chocolate consumption, compared with those in the lowest quartile, had a lower risk of developing GDM (OR 0.76, $95 \%$ CI $0.66,0.86 ; P$ for trend $<0.001$; Table 2). In model 2 with

(1)


Table 2. Risk for gestational diabetes mellitus associated with chocolate consumption before and during early pregnancy (Medians, odds ratios, ranges and $95 \%$ confidence intervals)

\begin{tabular}{|c|c|c|c|c|c|c|c|c|c|c|c|}
\hline & \multicolumn{2}{|c|}{ Q1 } & \multicolumn{2}{|c|}{ Q2 } & \multicolumn{2}{|c|}{ Q3 } & \multicolumn{2}{|c|}{ Q4 } & \multicolumn{2}{|c|}{$\begin{array}{l}\text { Every } 25 \mathrm{~g} / \mathrm{d} \\
\text { increment }\end{array}$} & \multirow[b]{2}{*}{$\begin{array}{l}P \text { for } \\
\text { trend }\end{array}$} \\
\hline & $\begin{array}{l}\text { Median or } \\
\text { OR }\end{array}$ & $\begin{array}{l}\text { Range or } \\
95 \% \mathrm{Cl}\end{array}$ & $\begin{array}{l}\text { Median or } \\
\text { OR }\end{array}$ & $\begin{array}{l}\text { Range or } \\
95 \% \mathrm{Cl}\end{array}$ & $\begin{array}{l}\text { Median or } \\
\text { OR }\end{array}$ & $\begin{array}{l}\text { Range or } \\
95 \% \mathrm{Cl}\end{array}$ & $\begin{array}{l}\text { Median or } \\
\text { OR }\end{array}$ & $\begin{array}{l}\text { Range or } \\
95 \% \mathrm{Cl}\end{array}$ & OR & $95 \% \mathrm{Cl}$ & \\
\hline $\begin{array}{l}\text { Median intake } \\
\text { (g/week) }\end{array}$ & 0 & $<5.82$ & $11 \cdot 7$ & $11 \cdot 7,18 \cdot 8$ & 37.5 & $37 \cdot 5,43 \cdot 7$ & 87.5 & $>56 \cdot 3$ & & & \\
\hline No. of cases & 563 & & 543 & & 407 & & 391 & & & & \\
\hline $\begin{array}{l}\text { No. of } \\
\text { participants }\end{array}$ & 21579 & & 23922 & & 19093 & & 20354 & & & & \\
\hline OR, model $1^{*}$ & 1.00 & & 0.88 & $0.78,0.99$ & 0.83 & $0.73,0.95$ & $0 \cdot 76$ & $0.66,0.8$ & 0.93 & $0.90,0.97$ & $<0.001$ \\
\hline OR, model 2† & 1.00 & & 0.88 & $0.78,0.99$ & 0.86 & $0.75,0.98$ & 0.77 & $0.67,0.88$ & 0.95 & $0.91,0.98$ & 0.002 \\
\hline OR, model 3‡ & 1.00 & & 0.88 & $0.78,1.00$ & 0.86 & $0.75,0.98$ & 0.78 & $0.67,0.90$ & 0.94 & $0.90,0.98$ & 0.002 \\
\hline
\end{tabular}

* Model 1: adjusted for age.

† Model 2: model 1 and further adjusted for BMI before pregnancy, depression, history of macrosomia babies, parity, smoking, drinking, education, occupation, household income and physical activity.

$\ddagger$ Model 3: model 2 and further adjusted for intakes of total meat, red meat, coffee, green tea, milk, soya isoflavone, Mg, dietary fibre, dietary fat, saturated fat, snacks (potato chips and other crackers) and total energy intake.

additional adjustment for BMI before pregnancy, depression, history of macrosomia babies, parity, smoking status, drinking status, education level, occupation, household income and physical activity, a similar result was observed (OR 0.77, $95 \%$ CI 0.67, 0.88; $P$ for trend =0.002). In model 3, further adjustment for dietary factors, including total meat, red meat, coffee, green tea, milk, soya isoflavone, $\mathrm{Mg}$, dietary fibre, dietary fat, saturated fat, snacks and total energy, did not materially alter the association either (OR 0.78, $95 \%$ CI 0.67, 0.90; $P$ for trend $=0.002$ ).

Table 3 shows the multivariable-adjusted OR of GDM for the highest quartile of chocolate consumption stratified by pre-pregnancy BMI, age, parity, smoking status and drinking status. Although relatively stronger associations were found in some subgroups, for example, in women with age $<35$ years and in current drinkers, the test for interaction showed that none of the above selected factors significantly modified the association (all $P$ for interaction $>0 \cdot 10$ ).

To test the robustness of the results, we conducted a sensitivity analysis restricted in 65166 women who reported that they have not substantially changed dietary habits during the past 5 years. In this restricted population, the OR of GDM for the highest quartile of chocolate consumption was 0.73 (95\% CI 0.62, $0 \cdot 87$ ), which was similar to the overall one. Repeated analyses using energy-adjusted data and using quintile for chocolate consumption yielded similar results (data not shown).

\section{Discussion}

The present study was, to our best knowledge, the first prospective cohort that examined the association between chocolate consumption and risk of GDM. Findings from our analyses suggested that higher chocolate consumption was associated with a significant lower risk of GDM even after controlling for important risk factors. In addition, the risk was not significantly affected by pre-pregnancy BMI, age, parity, smoking status or drinking status.

One possible mechanism underlying the inverse association of chocolate consumption with the risk of GDM may be related to improvement in insulin resistance. Several small randomised controlled trials (number of subjects ranging from 24 to 42 ) have reported an improvement in insulin resistance by cocoa product intervention (e.g. chocolate, cocoa drink $)^{(16-20)}$. Results from a subsequent trial conducted in ninety elderly individuals were in support of the previous trials by showing a significant improvement in homeostasis model assessment for insulin resistance, a common insulin resistance index, after 8 weeks of cocoa drink intervention ${ }^{(21)}$. In addition, fasting insulin was also significantly improved by cocoa intervention; however, fasting glucose appeared not to be affected ${ }^{(10)}$. Unfortunately, there are no randomised controlled trials examining the effects of chocolate consumption on glucose metabolism among pregnant women.

Although there was no previous study looking at the chocolate-GDM association, the association between chocolate consumption and risk of type 2 diabetes has been examined in five previous cohort studies ${ }^{(22-26)}$. Some, but not all, reported a significant lower risk of type 2 diabetes associated with chocolate consumption. A pooled analysis showed that higher chocolate consumption, compared with lower chocolate consumption, was associated with a $18 \%$ reduction in the risk of type 2 diabetes (pooled relative risk 0.82, $95 \%$ CI 0.70 , $0.96)^{(27)}$. In spite of different research outcomes, the result of the present study was generally consistent with the pooled result of previous studies in both direction and magnitude.

We detected an inverse relationship between chocolate consumption and risk of GDM; however, it should be applied within the range up to once per $d$ because less than $0.6 \%$ women of the total participants consumed chocolate more than once per $\mathrm{d}$. In an additional analysis, the fully adjusted OR was 1.22 (95\% CI 0.72 , $2 \cdot 08$ ) for women who had chocolate consumption more than once per d compared with those who had chocolate consumption less than once per month. In the Atherosclerosis Risk in Communities Study, consuming moderate amount of chocolate at 2-6 times/ week was associated with a lower risk of type 2 diabetes, while consuming more than once per $d$ yielded no significant result ${ }^{(26)}$. Similarly, in the Women's Health Initiative study, the association 
Table 3. Multivariable-adjusted risk of gestational diabetes mellitus for the highest quartile of chocolate consumption compared with the lowest quartile stratified by participant characteristics*

(Odds ratios and $95 \%$ confidence intervals)

\begin{tabular}{lrllc}
\hline & $\begin{array}{c}\text { Cases/ } \\
\text { participants }\end{array}$ & OR & $95 \% \mathrm{Cl}$ & $\begin{array}{c}P \text { for } \\
\text { interaction }\end{array}$ \\
\hline $\mathrm{BMl}<25 \mathrm{~kg} / \mathrm{m}^{2}$ & & & & \\
$\quad$ Yes & $1359 / 76093$ & 0.78 & $0.67,0.93$ & 0.36 \\
$\quad$ No & $545 / 8855$ & 0.73 & $0.55,0.98$ & \\
Age $<35$ years & & & & \\
$\quad$ Yes & $1136 / 64455$ & 0.69 & $0.55,0.82$ & 0.19 \\
$\quad$ No & $768 / 20493$ & 0.90 & $0.72,1.12$ & \\
Delivery history & & & & \\
$\quad$ Yes & $1060 / 47602$ & 0.82 & $0.68,0.99$ & 0.50 \\
$\quad$ No & $804 / 35244$ & 0.66 & $0.52,0.83$ & \\
Smoking status & & & & \\
$\quad$ Never smokers & $1062 / 49728$ & 0.88 & $0.73,1.07$ & 0.29 \\
$\quad$ Former smokers & $729 / 30749$ & 0.64 & $0.51,0.80$ & \\
$\quad$ Current smokers & $100 / 3875$ & 0.61 & $0.32,1.18$ & \\
Drinking status & & & & \\
$\quad$ Never drinkers & $734 / 30181$ & 0.81 & $0.65,1.03$ & 0.53 \\
$\quad$ Former drinkers & $1013 / 46900$ & 0.74 & $0.62,0.90$ & \\
$\quad$ Current drinkers & $151 / 7705$ & 0.58 & $0.31,0.96$ & \\
\hline
\end{tabular}

* Adjusted for age, BMI before pregnancy, depression, history of macrosomia babies, parity, smoking, drinking, education, occupation, household income, physical activity, and intakes of total meat, red meat, coffee, green tea, milk, soya isoflavone, Mg, dietary fibre, dietary fat, saturated fat, snacks (potato chips and other crackers) and total energy; interaction tests were conducted by entering the interaction terms in the logistical model.

between chocolate consumption and risk of type 2 diabetes was not dose dependent either, with an inverse association observed only at a moderate level of intake (i.e. <3 servings/week) in two subgroups of postmenopausal women ${ }^{(25)}$. Of note, because chocolate contains a high amount of sugar and fat, excessive intake may increase the risk of overweight and obesity and hence outweigh any potential benefits on health.

The main strengths of our study included a large sample size and a prospective cohort design. In addition, data on GDM were obtained from medical records but not from self-report questionnaire, which decreased misclassification bias. However, several limitations of the present study also merited consideration. First, the short duration between baseline assessment and outcome incidence may increase the risk of reverse causation. Although women with a history of GDM were excluded from the analysis, those at high risk of GDM (e.g. with BMI $>25 \mathrm{~kg} / \mathrm{m}^{2}$ ) were likely to have lower chocolate consumption because of its high content of sugar and fat, which might result in an overestimated association. Yet subgroup analysis by BMI showed similar results as shown in Table 3, indicating a low risk of such bias. Second, fat, sugar and polyphenol contents vary by chocolate types, but data on types of chocolate, as with previous cohort studies on chocolate research ${ }^{(27)}$, were not available. As a result, any comparison on the risk of GDM between dark, milk and white chocolate could not be performed, and whether the observed association was attributed to cocoa polyphenol could not be tested either. However, milk chocolate appeared to be the most consumed chocolate in Japan ${ }^{(28)}$. Third, as shown in Table 1, higher chocolate consumption was associated with other factors, including higher consumptions of coffee and green tea, which were associated with lower risk of type 2 diabetes ${ }^{(29)}$.
Although they were statistically adjusted, the influences of residual confounding could not be ruled out because of the observational nature of the study. For instance, we could not adjust for family history of diabetes as well as other unmeasured factors. Fourth, the incidence of GDM was relatively lower. One explanation may be that women participating in our study were more health conscious, which might lead to a limited generalisability of the present. Finally, a large sample size that could make minuscule differences between groups becomes statistically significant. Due to the above limitations, the findings of the present study should be treated with caution.

In conclusion, our study provided the first evidence that higher chocolate consumption was associated with a lower risk of GDM. This finding needed to be confirmed in further well-designed cohort studies among other population as well as randomised controlled trials taking GDM as the endpoint.

\section{Acknowledgements}

We thank all the participants and staff involved in the Japan Environment and Children's Study. Members of the Japan Environment and Children's Study as of 2018 (principal investigator, Toshihiro Kawamoto): Yukihiro Ohya (National Center for Child Health and Development, Tokyo, Japan), Reiko Kishi (Hokkaido University, Sapporo, Japan), Nobuo Yaegashi (Tohoku University, Sendai, Japan), Koichi Hashimoto (Fukushima Medical University, Fukushima, Japan), Chisato Mori (Chiba University, Chiba, Japan), Shuichi Ito (Yokohama City University, Yokohama, Japan), Zentaro Yamagata (University of Yamanashi, Chuo, Japan), Hidekuni Inadera (University of Toyama, Toyama, Japan), Michihiro Kamijima (Nagoya City University, Nagoya, Japan), Takeo Nakayama (Kyoto University, Kyoto, Japan), Hiroyasu Iso (Osaka University, Suita, Japan), Masayuki Shima (Hyogo College of Medicine, Nishinomiya, Japan), Yasuaki Hirooka (Tottori University, Yonago, Japan), Narufumi Suganuma (Kochi University, Nankoku, Japan), Koichi Kusuhara (University of Occupational and Environmental Health, Kitakyushu, Japan) and Takahiko Katoh (Kumamoto University, Kumamoto, Japan).

The Japan Environment and Children's Study was funded by the Ministry of the Environment, Japan. This work was partly supported by Japan Society for the Promotion of Science KAKENHI (A18H06391, T19K214700) to J.-Y. D.

J.-Y. D. designed the study, analysed the data and wrote the manuscript. T. K., S. I. and H. I. conducted the technique review and reviewed and edited the manuscript. M. C., Y. K., K. Y. and K. U. reviewed the manuscript. T. K., S. I., K. U. and H. I. helped to collect the data. J.-Y. D. is the guarantor of this work and, as such, had full access to all the data in the study and takes responsibility for the integrity of the data and the accuracy of the data analysis.

The authors have no conflicts of interest to report.

The funders had no role in the design and conduct of the study; collection, management, analysis and interpretation of the data; preparation, review or approval of the manuscript; or the decision to submit the manuscript for publication. 


\section{References}

1. American Diabetes Association (2004) Gestational diabetes mellitus. Diabetes Care 27, S88-S90.

2. Reece EA, Leguizamon G \& Wiznitzer A (2009) Gestational diabetes: the need for a common ground. Lancet 373, 1789-1797.

3. Bellamy L, Casas J-P, Hingorani AD, et al. (2009) Type 2 diabetes mellitus after gestational diabetes: a systematic review and meta-analysis. Lancet 373, 1773-1779.

4. Song C, Lyu Y, Li C, et al. (2018) Long-term risk of diabetes in women at varying durations after gestational diabetes: a systematic review and meta-analysis with more than 2 million women. Obes Rev 19, 421-429.

5. Tobias DK, Stuart JJ, Li S, et al. (2017) Association of history of gestational diabetes with long-term cardiovascular disease risk in a large prospective cohort of US women. JAMA Intern Med 177, 1735-1742.

6. Boerschmann H, Pfluger M, Henneberger L, et al. (2010) Prevalence and predictors of overweight and insulin resistance in offspring of mothers with gestational diabetes mellitus. Diabetes Care 33, 1845-1849.

7. Maftei O, Whitrow MJ, Davies MJ, et al. (2015) Maternal body size prior to pregnancy, gestational diabetes and weight gain: associations with insulin resistance in children at 9-10 years. Diabet Med 32, 174-180.

8. Ried K, Fakler P \& Stocks NP (2017) Effect of cocoa on blood pressure. Cochrane Database Syst Rev, issue 4, CD008893.

9. Hooper L, Kay C, Abdelhamid A, et al. (2012) Effects of chocolate, cocoa, and flavan-3-ols on cardiovascular health: a systematic review and meta-analysis of randomized trials. Am J Clin Nutr 95, 740-751.

10. Lin X, Zhang I, Li A, et al. (2016) Cocoa flavanol intake and biomarkers for cardiometabolic health: a systematic review and meta-analysis of randomized controlled trials. $J$ Nutr 146, 2325-2333.

11. Veronese N, Demurtas J, Celotto S, et al. (2019) Is chocolate consumption associated with health outcomes? An umbrella review of systematic reviews and meta-analyses. Clin Nutr 38, 1101-1108

12. Kawamoto T, Nitta H, Murata K, et al. (2014) Rationale and study design of the Japan Environment and Children's Study (JECS). BMC Public Health 14, 25.

13. Michikawa T, Nitta H, Nakayama SF, et al. (2018) Baseline profile of participants in the Japan Environment and Children's Study (JECS). J Epidemiol 28, 99-104.

14. Yokoyama Y, Takachi R, Ishihara J, et al. (2016) Validity of short and long self-administered food frequency questionnaires in ranking dietary intake in middle-aged and elderly Japanese in the Japan Public Health Center-Based Prospective Study for the Next Generation (JPHC-NEXT) protocol area. J Epidemiol 26, 420-432.

15. Hiramatsu Y, Haneda M, Yasuhi I, et al. (2015) The joint committee with the Japan Society of Diabetes and Pregnancy and the Japan Diabetes Society 'An Abnormal Glucose
Metabolism During Pregnancy and the Standardization of Its Diagnostic Criteria'. JJapan Diabetes Soc 58, 801-803.

16. Davison K, Coates AM, Buckley JD, et al. (2008) Effect of cocoa flavanols and exercise on cardiometabolic risk factors in overweight and obese subjects. Int J Obes 32, 1289-1296.

17. Grassi D, Desideri G, Necozione S, et al. (2008) Blood pressure is reduced and insulin sensitivity increased in glucose-intolerant, hypertensive subjects after 15 days of consuming highpolyphenol dark chocolate. J Nutr 138, 1671-1676.

18. Grassi D, Lippi C, Necozione S, et al. (2005) Short-term administration of dark chocolate is followed by a significant increase in insulin sensitivity and a decrease in blood pressure in healthy persons. Am J Clin Nutr 81, 611-614.

19. West SG, McIntyre MD, Piotrowski MJ, et al. (2014) Effects of dark chocolate and cocoa consumption on endothelial function and arterial stiffness in overweight adults. Br J Nutr 111, 653-661.

20. Almoosawi S, Tsang C, Ostertag LM, et al. (2012) Differential effect of polyphenol-rich dark chocolate on biomarkers of glucose metabolism and cardiovascular risk factors in healthy, overweight and obese subjects: a randomized clinical trial. Food Funct 3, 1035-1043.

21. Mastroiacovo D, Kwik-Uribe C, Grassi D, et al. (2015) Cocoa flavanol consumption improves cognitive function, blood pressure control, and metabolic profile in elderly subjects: the Cocoa, Cognition, and Aging (CoCoA) Study - a randomized controlled trial. Am J Clin Nutr 101, 538-548.

22. Oba S, Nagata C, Nakamura K, et al. (2010) Consumption of coffee, green tea, oolong tea, black tea, chocolate snacks and the caffeine content in relation to risk of diabetes in Japanese men and women. Br J Nutr 103, 453-459.

23. Matsumoto C, Petrone AB, Sesso HD, et al. (2015) Chocolate consumption and risk of diabetes mellitus in the Physicians' Health Study. Am J Clin Nutr 101, 362-367.

24. Crichton GE, Elias MF, Dearborn P, et al. (2017) Habitual chocolate intake and type 2 diabetes mellitus in the MaineSyracuse Longitudinal Study: (1975-2010): prospective observations. Appetite 108, 263-269.

25. Greenberg JA, Manson JE, Tinker L, et al. (2017) Chocolate intake and diabetes risk in postmenopausal American women. Eur J Clin Nutr 71, 1088-1093.

26. Greenberg JA (2015) Chocolate intake and diabetes risk. Clin Nutr 34, 129-133.

27. Yuan S, LiX, Jin Y, et al. (2017) Chocolate consumption and risk of coronary heart disease, stroke, and diabetes: a meta-analysis of prospective studies. Nutrients $\mathbf{9}, 688$.

28. Chocolate online survey from MyVoice Communications, Inc. = https://myel.myvoice.jp/products/detail.php?product_id=16102 (accessed June 2019) (in Japanese).

29. Iso H, Date C, Wakai K, et al. (2006) The relationship between green tea and total caffeine intake and risk for self-reported type 2 diabetes among Japanese adults. Ann Intern Med 144, 554-562. 